CORRIGENDUM

doi:10.1038/nature10604

\title{
DNA demethylation for hormone-induced transcriptional derepression
}

Mi-sun Kim, Takeshi Kondo, Ichiro Takada, Min-young Youn, Yoko Yamamoto, Sayuri Takahashi, Takahiro Matsumoto, Sally Fujiyama, Yuko Shirode, Ikuko Yamaoka, Hirochika Kitagawa, Ken-ichi Takeyama, Hiroshi Shibuya, Fumiaki Ohtake \& Shigeaki Kato

Nature 461, 1007-1012 (2009)

Several lanes of the ChIP analyses in this Letter were inadvertently duplicated or erroneously created during figure assembly. We now provide corrected figure panels for Figs $1 \mathrm{f}, 2 \mathrm{c}, 2 \mathrm{f}, 2 \mathrm{~g}$ and $3 \mathrm{~h}$ and Supplementary Figs S8, S9a, S9b, S11, S13b, S18 and S28. Our results and conclusions are not affected by these errors, but we apologise for the careless mistakes made.

During initial preparation of the figure panels, the panels for the negative controls (with no obvious signals), inputs and some data were inappropriately duplicated and mixed up. The experiments were performed several times, so a set of data with adequate negative controls and inputs from one experimental round could be found. We have replaced the previous set of data in these panels with a correct set, and confirmed that these corrections do not affect the original claims in the published text. Representative ChIP data are displayed and significance was statistically assessed from several independently repeated experiments. We have also repeated the experiments and obtained the same results as those in the published figures.

In Fig. 1f, lane 5 of anti-AcH3, lane 1 of anti-5-MetC, and the antiIgG panel of a previous data set were left blank without placing of gel images. In Fig. $2 c$, the panels of distal regions $(-3,907 /-3,442$; left panels) and the input panels $(-230 /+130)$ were duplicated. The predicted data of anti-5MetC was inadvertently mixed up. In Figs $2 f, 2 g$ and $3 \mathrm{~h}$, the input panels were duplicated.

Further errors in the Supplementary Figures of the original Letter are described and corrected in the Supplementary Information of this Corrigendum, and the supporting raw data is also presented.

Supplementary Information is linked to the online version of this Corrigendum at www.nature.com/nature.
$1 f$

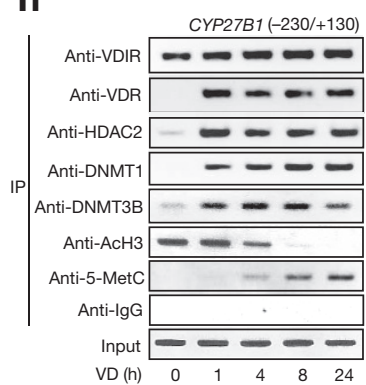

2c

Anti-MeCP

Anti-MBD

Anti-MBD2

Anti-MBD3

Anti-MBD4

IP

Anti-5-MetC

Anti-VDR

Anti-HDAC2

Anti-DNMT1

Anti-DNMT3B

Anti-lgG

Input

VD $(24 h)$

PTH (1 h)

CYP27B1

CYP27B1

$(-3907 /-3442) \quad(-230 /+130)$

$2 f$

CYP27B1 $(-230 /+130)$

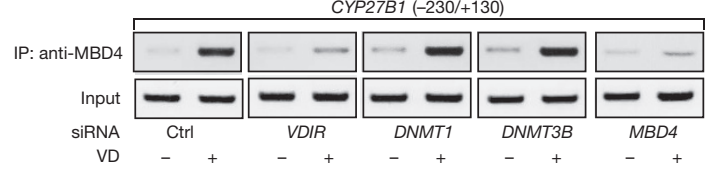

2g

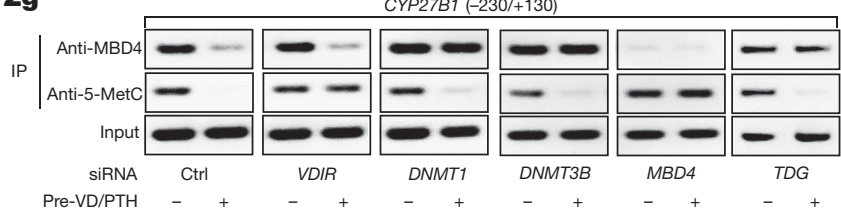

3h

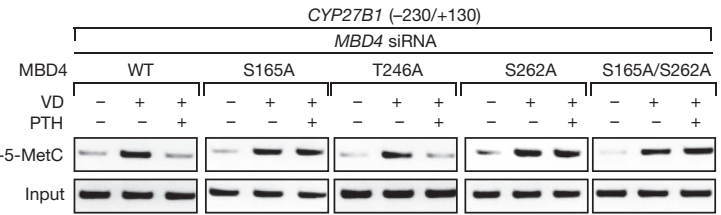

Figure $1 \mid$ Corrected versions of Figs 1f, 2c, 2f, $2 \mathrm{~g}$ and $3 \mathrm{~h}$. 